\title{
CORRECTIONS
}

\section{US doctors are unwilling to pay more to abolish industry funded education}

The eighth paragraph of this news article by Bob Roehr stated that "most doctors $(83 \%)$ believed that industry support should be eliminated from CME [continuing medical education]" (BMJ 2011;342:d2948, doi:10.1136/bmj.d2948). This is wrong. In fact, the study from which he was citing found that only $17 \%$ of doctors believed this.

Cite this as: $B M J 2011 ; 342: \mathrm{d} 3888$ 